\title{
IDENTIFYING HUMAN USING EAR IMAGING WITH MACHINE LEARNING TECHNIQUES
}

\author{
RAHUL LAHKAR \\ Department of Information Technology, \\ Gauhati University, Guwahati, Assam, 781014, India \\ rahul@gauhati.ac.in \\ KHURSHID ALAM BORBORA \\ Department of Computer Science, \\ IDOL- Gauhati University, Guwahati, Assam, 781014, India \\ khurshidborbora007@yahoo.co.in
}

\begin{abstract}
In order to identify a person based on some physiological attributes or behavioral characteristics various biometrics have been introduced so far which includes face, retina, fingerprints etc. But research has revealed that the human ear also possesses some unique features which can be used as a biometric for recognition of humans. This paper uses the ear as a biometric to identify human while comparing the performance analysis of dimensionality reduction techniques PCA and LDA with various classification techniques.
\end{abstract}

Keywords: Ear; Eigen Ear; Ear Biometrics; PCA; LDA; Recognition; Identification.

\section{Introduction}

To identify a human there are several systems available which use the unique features of some physiological attributes that some parts of the human body possesses which include the face, fingerprints, retina etc. In contrast to this, it has been discovered that the human ear too carries promising physiological attributes and anthropologists have proven [5] the uniqueness of human ear which claims the possession of unique attributes that can be used as a biometric for human recognition. Actually, Ear as a biometric, has several advantages over the others in the concrete. First of all, it is a biometric without contact as similar to face but face biometric suffers from various expressions of the face which is not possible in case of ear. Secondly, ear biometric can give outstanding performance in identifying human when only one side of a face is available. In the situations where the face may have occlusion, the ear can be a useful biometric for human identification. Lastly, the size of the ear as compared to other biometric-iris, retina and fingerprint is larger, and thus its detection becomes easier. Many researches have been inferring the use of ear as biometric is conspicuous and outstanding.

The external anatomy of the human ear is shown in Figure 1. The composition of the external human ear includes the helix or the outer rim, the anti-helix which is parallel to the helix but it resides inside the lobe, and the very distinct $\mathrm{u}$-shaped notch which is known as intertragus notch. The upper shape of the ear also includes triangular fossa and scaphoid fossa. Fig 1 shows the other parts like cavum concha, tragus etc.

In this paper, we apply the concept of dimension reduction techniques such as Principal Components Analysis (PCA) and Linear Discriminant Analysis (LDA), which decompose the high dimensional ear images into low dimensional space. In PCA, we find the principal components by extracting the eigen ears from the ear images of the training set which hold the highest information of the ear as required. Test images are mapped with the training images as one-to-many using eigen ears and various classifiers to identify the correct ear. In LDA, projection vectors are computed which also reduces the ear dimensions from high to low. All the projected ear images form the maximum between-class scatter and minimum within-class scatter. The experiments are performed on the IITD Ear database and a comparative study is done on ear recognition using PCA and LDA. 


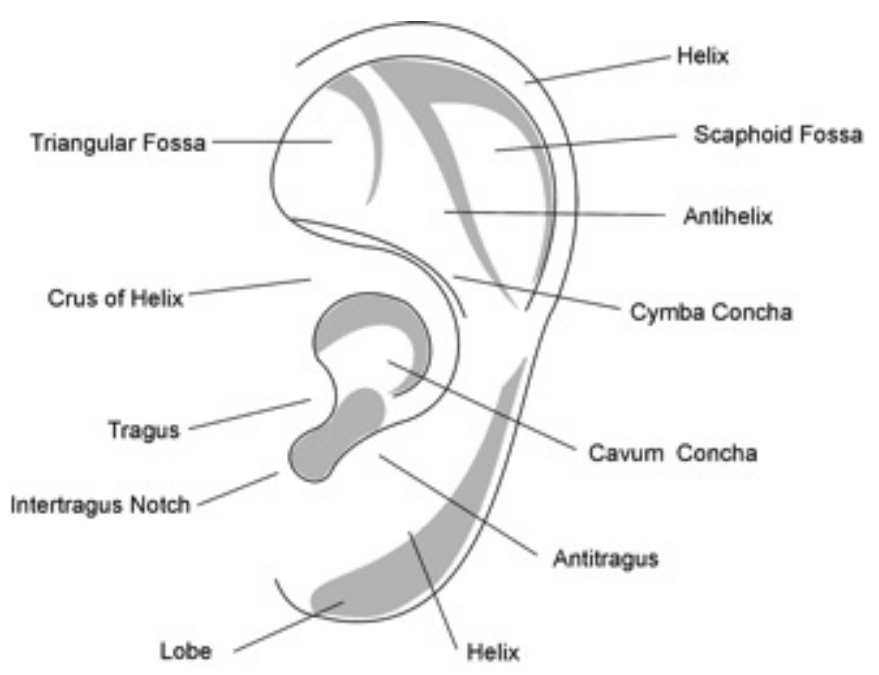

Fig 1. The external ear [5]

\section{Literature Survey}

If we take a voyage back to the history of Ear Biometric then it is found that in the early 1880 s, a French police officer named Alphonse Bertillon observed the prospective of using several body parts of the human which includes the ear also as a tool of identification and he termed this as Anthropometry [1]. A doctor in Prague in 1906 , named R. Imhofer appealed that only four features were important enough to discriminate a set containing 500 ears [2]. Some researchers used ear pictures of new born babies to find their identity and got satisfied with the morphological constancy of the ear [3]. In the year 1989, more than 10,000 ears were inspected by Iannarelli [4] and developed an anthropometric methodology for ear identification which is appraised as one of the distinguished works in this field.

Comparative summary of the ear recognition techniques and other related works [5] is presented in a tabular form in Table1. The column Methodology provides a short description of the techniques or methods used by the authors and the datasets used for testing and reported performances are also stated in Table 1.

Table 1. Ear recognition related works

\begin{tabular}{|c|c|c|c|}
\hline Researcher & Methodology & Dataset & $\begin{array}{c}\text { Performance } \\
\text { \% }\end{array}$ \\
\hline $\begin{array}{c}\text { Moreno et } \\
\text { al. }\end{array}$ & $\begin{array}{c}\text { Geometric features like shape, points, } \\
\text { wrinkles }\end{array}$ & Own & 43 \\
\hline Mu et al. & Structure and shape of the ear as features & USTB II & 85 \\
\hline Chang et al. & Principal Component Analysis (PCA) & UND E & 71.6 \\
\hline Victor et al. & Principal Component Analysis (PCA) & Own (images & Nearly 100 \\
\hline Yuizono & Genetic Algorithms & Own & 99.2 \\
\hline Hurley et al. & Force field feature extraction with PCA & NIST & 84.3 and 91.2 \\
\hline $\begin{array}{c}\text { Hurley and } \\
\text { Carter }\end{array}$ & $\begin{array}{c}\text { Chape of outer ear with Neural } \\
\text { Classifiers }\end{array}$ & UND E & 84 \\
\hline $\begin{array}{c}\text { L Nanni et } \\
\text { al. }\end{array}$ & Color space fusion with Gabor Filters \\
\hline $\begin{array}{c}\text { S Prakash et } \\
\text { al. }\end{array}$ & $\begin{array}{c}\text { Edge detection using graph-based } \\
\text { concept }\end{array}$ & UND-J2 & 99 \\
\hline Kumar et al. & Orthogonal log-Gabor filters & IITD & 95.93 \\
\hline Islam et al. & $\begin{array}{c}\text { Combination of ear and frontal face } \\
\text { (Neutral facial expression) }\end{array}$ & UND-FRGC & 99 \\
\hline $\begin{array}{c}\text { Arbab-Zavar } \\
\text { et al. }\end{array}$ & $\begin{array}{c}\text { Hybrid method with wavelet-based } \\
\text { analysis for part of the ear recognition } \\
\text { using PCA and RPCA }\end{array}$ & Extended M2VTS & $\begin{array}{c}98.4 \text { (without } \\
\text { occlusion) } \\
68.9 \\
\text { (Occlusion) }\end{array}$ \\
\hline
\end{tabular}




\begin{tabular}{|c|c|c|c|}
\hline Chan et al. & $\begin{array}{c}\text { Quaternionic quadrature filter and the } \\
\text { Quaternionic Code }\end{array}$ & UND and IITD & $\begin{array}{c}95.73,94.72 \\
\text { and } 87.88\end{array}$ \\
\hline $\begin{array}{c}\text { Yahui Liu et } \\
\text { al. }\end{array}$ & Ear-parotic face angle & Own & NA \\
\hline Abaza et al. & $\begin{array}{c}\text { Local ternary patterns (LTP) and local } \\
\text { binary patterns (LBP) }\end{array}$ & 80.68 and 68.18 \\
\hline $\begin{array}{c}\text { K } \\
\text { Annpurani } \\
\text { et al. }\end{array}$ & $\begin{array}{c}\text { Fusion of shape of ear with shape of } \\
\text { Tragus }\end{array}$ & AMI and IITD & 99.9 and 100 \\
\hline Dinkar et al. & Pattern recognition using ANN & Own & 94 \\
\hline
\end{tabular}

\section{Experiments}

Our work is divided in two parts: (i) for extracting the features of an ear image by using dimensionality reduction techniques PCA (Principal Components Analysis) and LDA (Linear Discriminant Analysis) and (ii) for classification purpose various classifiers are used and results are analyzed which is discussed in the later part of this paper. The dataset used is the IITD Ear database [6]. It is a collection of images of the students and staffs at Indian Institute of Technology, Delhi, India, of age group 14-58 years. The resolution of the ear images are $272 \times$ 204 pixels and available in '.bmp' format. The experimented version of the database consists of 493 raw images from 125 persons having 3 to 6 samples of an individual. In addition to these images the database also provides automatically normalized and cropped ear images. Recently, a larger version of ear database from 221 users with 793 processed and nicely cropped ear images is added to it and it is used for our experiments. This database may be a suitable database for experiments to be done with images with occlusion and jewelry.

The Fig 2(a) shows some of the randomly chosen ear images of different individuals and Fig 2(b) shows some of the random images from the database which are processed cropped and aligned. We carried out the experiments on these pre-processed images.
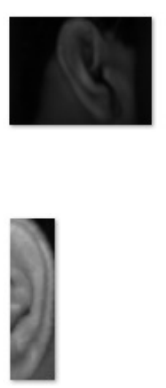
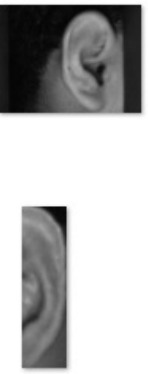

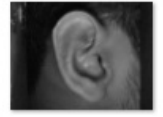

(a)

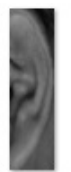

(b)
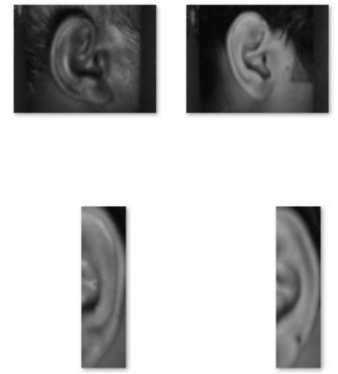

Fig 2. Sample images from IITD dataset

\subsection{Experiment -1: Using PCA}

Principal components analysis is a popular linear subspace unsupervised approach for deriving $h$-low-dimensional set of features from a large set of variables of $d$-dimensionality with $h<d$. The low dimension space is called as 'Eigen space', which is defined by a set of 'Eigen vectors' of dataset to be used.

For an ear image $\Gamma(P \times Q)$, where $\mathrm{P}$ and $\mathrm{Q}$ are the width and height of the image respectively. The Image is first transformed into a vector of length $P \times Q$. The feature matrix of $Z$ training ear images in a dataset is then given by $\Gamma=\left[\Gamma_{1}, \Gamma_{2}, \ldots, \Gamma_{Z}\right]$, and the mean of the training set is calculated as shown in Eq. 1-

$$
\Psi=\frac{1}{\mathrm{z}} \sum \Gamma_{\mathrm{i}}
$$

The mean is subtracted, i.e. $\phi_{\mathrm{i}}=\Gamma_{\mathrm{i}}-\Psi$, and the data matrix created as in Eq. 2-

$$
A=\left[\phi_{1}, \phi_{2}, \ldots, \phi_{\mathrm{z}}\right]((P \times Q) \times Z)
$$

Next, the covariance matrix of $\mathrm{A}$ is then calculated as shown in Eq. 3-

$$
C=A A^{T}
$$

After finding $C$, the eigen-values $\lambda_{z}$ and eigenvectors $V_{z}$ of $C$ are calculated and the eigenvectors are arranged and stored according to the corresponding eigen-values. Dimensionality reduction is then obtained by keeping only the top $n$ eigen-vectors which yields a projection matrix P. For an ear image $\mathbf{X}$, which is of the same size as the images in training set, is first mean-normalized by $\phi_{\mathrm{x}}=X-\Psi$, and then transformed into Eigen-ear components, i.e. projected into ear space, by $\omega=P^{X} \phi_{\mathrm{x}}$. 


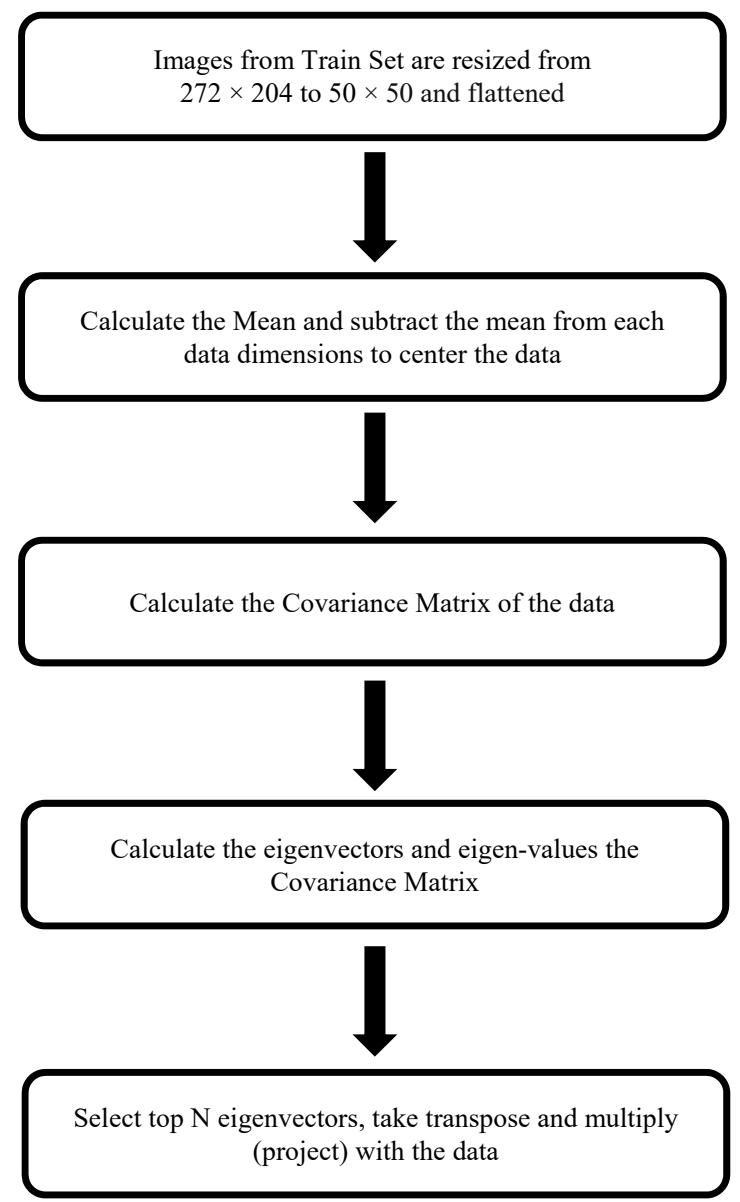

Fig 3. Work-flow of the experiment using PCA

The work-flow of implementing PCA on our experiment is shown in fig 3. These selected top N Eigen vectors are considered as the principal components after projection of which on the data yields the Eigen Ears. These Eigen Ears which contain less but important features are used for classification purpose from the testing set data. The performance of recognition is observed by using various classifiers such as K- Nearest Neighbor (KNN), Logistic regression (LR), Gaussian Naïve Bayes (GNB), Support Vector Machines (SVM) and Decision Tree (DT) and the results are discussed in later section.

In fig 4- images (3), (4), (5) are showing the results of PCA (Eigen Ears).

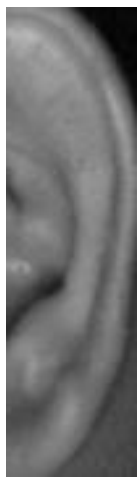

(1)

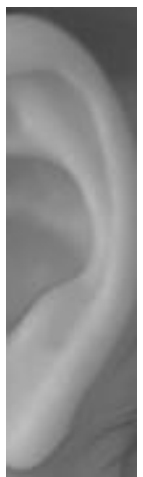

(2)

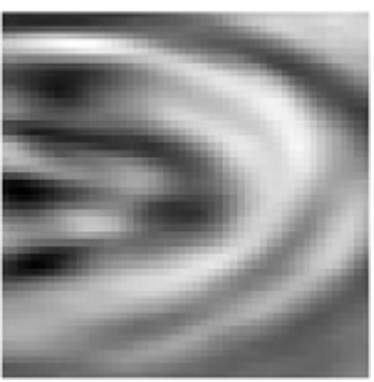

(3)

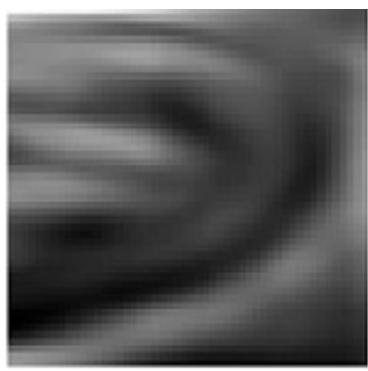

(4)

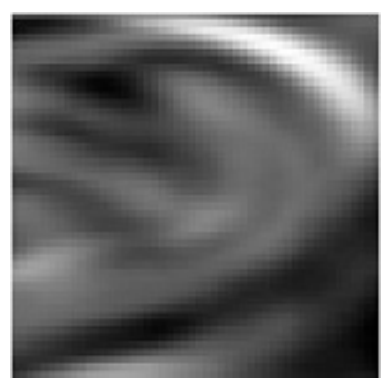

(5)

Fig 4. Sample processed images (1), (2) from IITD database, few Eigen ear images (3), (4), (5) of dimension $50 \times 50$. 


\subsection{Experiment -2: Using LDA}

Linear Discriminant Analysis (LDA) also known as Fischer Discriminants [7] is a common technique used for dimensionality reduction and classification in the preprocessing steps for various machine learning and pattern recognition applications. Steps involved in LDA are as follows:

a) First of all, the $m$-dimensional mean vectors for the different classes in the dataset are computed.

b) Next step is to find the in-between-class and within-class scatter matrices.

c) After this, eigenvectors $\left(\boldsymbol{e}_{1}, \boldsymbol{e}_{2}, \ldots, \boldsymbol{e}_{\mathrm{m}}\right)$ and their corresponding eigenvalues $\left(\boldsymbol{\lambda}_{1}, \boldsymbol{\lambda}_{2}, \ldots ., \lambda_{\mathrm{m}}\right)$ for all the scatter matrices are computed and eigenvectors are sorted by decreasing order of corresponding eigenvalues.

d) From this $\boldsymbol{k}$ eigen-vectors having largest eigenvalues are chosen to form $\boldsymbol{m} \times \boldsymbol{k}$ dimensional matrix (M) where every column is an eigenvector.

e) This $\boldsymbol{m} \times \boldsymbol{k}$ dimensional matrix is used to transform the samples onto the new subspace as: $\mathbf{Y}=\mathbf{X} \times \mathbf{M}$, where $\mathbf{X}$ is the sample $\boldsymbol{n} \times \boldsymbol{m}$ dimensional data matrix, Y is the transformed $\boldsymbol{n} \times \boldsymbol{k}$ dimensional matrix in the new subspace.

After applying LDA technique, various classifiers such as KNN, LR, GNB, SVM and DT are implemented for recognition purpose as we did in case of PCA and the outcomes are discussed in results section.

\section{Results and Discussion}

After applying PCA, the rate of accuracies is shown by increasing the number of Eigen ears as well as the number of training samples.

Table 2. Observed accuracy with one train sample

\begin{tabular}{|c|c|c|}
\hline Train Samples & Eigen ears & Accuracy \\
\hline 1 & 5 & 0.2954 \\
\hline 1 & 10 & 0.5045 \\
\hline 1 & 15 & 0.5909 \\
\hline 1 & 20 & 0.6409 \\
\hline 1 & 30 & 0.6688 \\
\hline 1 & 40 & 0.6818 \\
\hline 1 & 50 & 0.6818 \\
\hline 1 & 60 & 0.6954 \\
\hline 1 & 90 & 0.7045 \\
\hline 1 & 100 & 0.7136 \\
\hline
\end{tabular}

In Table 2, only one training sample of an individual is taken and the effect on recognition rate is observed by increasing the number of Eigen Ears.

Table 3. Observed accuracy with two train samples

\begin{tabular}{|c|c|c|}
\hline Train Samples & Eigen ears & Accuracy \\
\hline 2 & 5 & 0.3409 \\
\hline 2 & 7 & 0.50 \\
\hline 2 & 9 & 0.60 \\
\hline 2 & 11 & 0.6272 \\
\hline 2 & 15 & 0.7090 \\
\hline 2 & 20 & 0.7409 \\
\hline 2 & 25 & 0.7590 \\
\hline 2 & 30 & 0.7772 \\
\hline 2 & 40 & 0.7909 \\
\hline 2 & 60 & 0.80 \\
\hline 2 & 70 & 0.8045 \\
\hline 2 & 80 & 0.8045 \\
\hline 2 & 90 & 0.8045 \\
\hline
\end{tabular}

In Table 3, there are two training samples of an individual and the effect on recognition rate is observed by increasing the number of Eigen Ears. We can observe the accuracy enhancement in lesser dimensions than of Table 1. 
Table 4. Observed accuracy with three train samples

\begin{tabular}{|c|c|c|}
\hline Train Samples & Eigen ears & Accuracy \\
\hline 3 & 5 & 0.8090 \\
\hline 3 & 7 & 0.8772 \\
\hline 3 & 9 & 0.8954 \\
\hline 3 & 11 & 0.9090 \\
\hline 3 & 15 & 0.9363 \\
\hline 3 & 20 & 0.9363 \\
\hline 3 & 25 & 0.95 \\
\hline 3 & 28 & 0.9545 \\
\hline 3 & 30 & 0.9545 \\
\hline 3 & 40 & 0.9545 \\
\hline 3 & 50 & 0.9545 \\
\hline
\end{tabular}

In Table 4, three training samples of an individual are taken and the effect on recognition rate is observed by increasing the number of Eigen Ears. We can achieve higher accuracy rate in very lesser dimensions than Table 2 and Table 3.

Graphically it is shown is Fig 5 where accuracies (Y-axis) are plotted against number of Eigen Ears (X-axis) for all the training sample variations. We can see that the maximum accuracy is achieved with three training samples but with 28 Eigen Ears only.

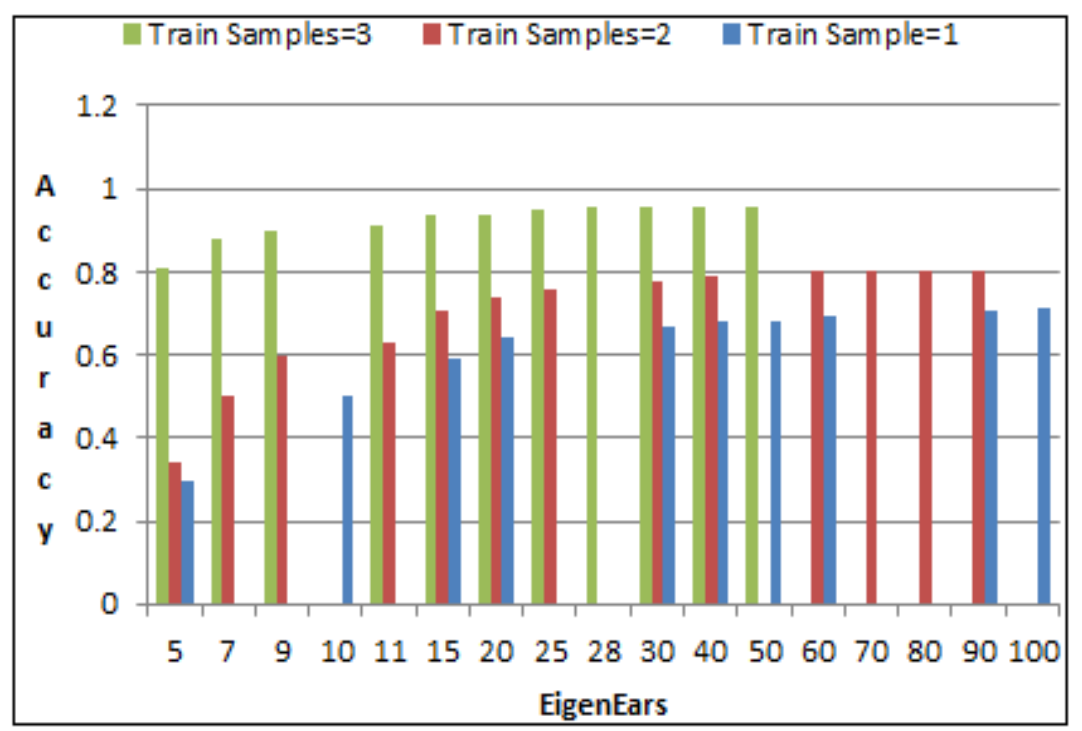

Fig 5. Accuracy vs. Eigenears with varying train samples

Table 5 lists the accuracies found when PCA is applied and Table 6 lists the accuracies found when LDA is applied. The accuracy results are plotted in Fig 6 for comparison.

Table 5: Results of PCA with various classifiers

\begin{tabular}{|c|c|c|c|c|}
\hline Classifier & Precision & Recall & f1-score & Mean Accuracy \\
\hline LR & 0.93 & 0.95 & 0.94 & 0.9511 \\
\hline KNN & 0.93 & 0.93 & 0.93 & 0.9436 \\
\hline SVM & 0.94 & 0.94 & 0.93 & 0.9398 \\
\hline GNB & 0.71 & 0.70 & 0.69 & 0.6992 \\
\hline DT & 0.39 & 0.45 & 0.40 & 0.4473 \\
\hline
\end{tabular}


Table 6: Results of LDA with various classifiers

\begin{tabular}{|c|c|c|c|c|}
\hline Classifier & Precision & Recall & f1-score & Mean Accuracy \\
\hline LR & 0.94 & 0.95 & 0.94 & 0.9511 \\
\hline KNN & 0.93 & 0.95 & 0.93 & 0.9473 \\
\hline SVM & 0.95 & 0.95 & 0.94 & 0.9548 \\
\hline GNB & 0.67 & 0.67 & 0.65 & 0.6654 \\
\hline DT & 0.57 & 0.62 & 0.57 & 0.6240 \\
\hline
\end{tabular}

The accuracies of PCA and LDA with various classifiers are compared and are shown graphically in figure 6.

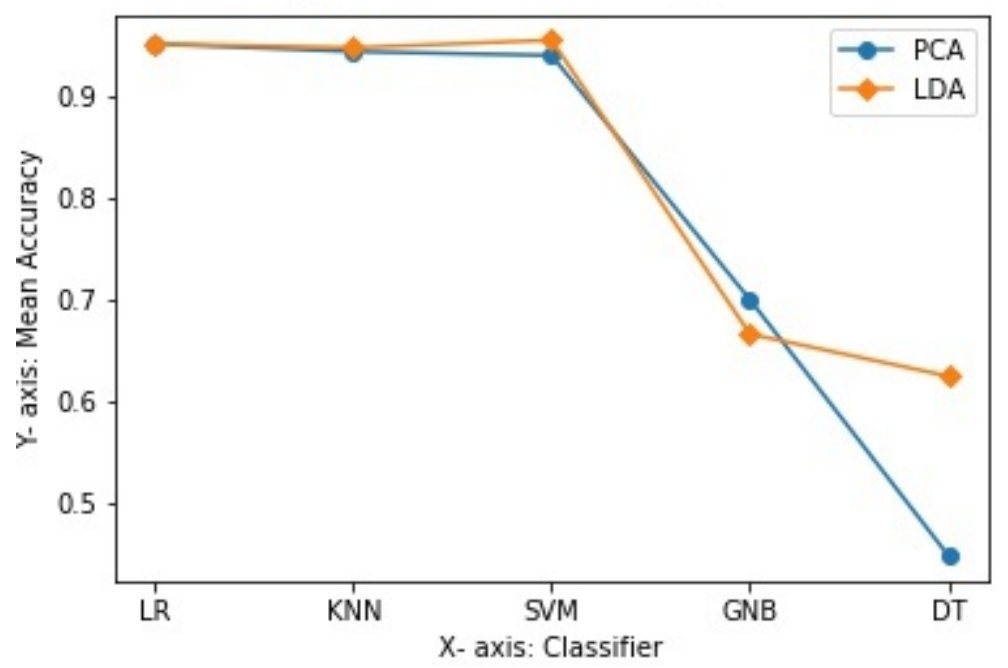

Fig 6. The mean accuracies of both PCA and LDA

\section{Conclusion}

In this paper, we have presented a comparative study of two dimensions reduction techniques the PCA and the LDA. The results of experiment- 1 are shown in table 5 and results of experiment- 2 are shown in table 6 and both the results are compared graphically in fig 6 .

In PCA, we have explored Eigen Ears and its effectiveness on increasing the number of train samples. We can infer that a greater number of train images would result in more accuracy with lesser dimensions. That means higher accuracy rate can be achieved with lesser Eigen Ears if we increase the number of train samples of each individual. There are several research articles which state the comparison of these two dimensions reduction techniques. Some find PCA is superior to LDA whereas some conclude LDA is superior to PCA depending on their experimental results. From the above results we now can compare the effectiveness of both the dimension reduction techniques PCA and LDA. We found that in three of the cases that is KNN, SVM and DT classifier, the results including the precision, recall and mean accuracy of LDA are improved over PCA and in one case that is, LR classifier, it is nearly equivalent. So, it can be inferred that LDA is superior dimension reduction technique in comparison to PCA which we have found experimentally in our methodology.

\section{References}

[1] Bertillon A, (1885): Identification anthropométrique: instructions signalétiques, 1885.

[2] "Die Bedeutung der Ohrmuschel für die Feststellung der Identität," Archiv für die Kriminologie, (1906), vol. 26, pp. 150-163.

[3] Fields C. et al., (1960): “The Ear of the Newborn as an Identification Constant,” Obstetrics and Gynecology, July 1960, pp. 98-102.

[4] Iannarelli A.(1989): "Ear identification, forensic identification series", Paramount Publishing Company.

[5] Lahkar R, Borbora K A (2019): "Human Identification using Ear as a Biometric: A Review", Journal of Artificial Intelligence Research \& Advances; 6(1): $99104 \mathrm{p}$.

[6] Kumar A and Wu C (2012), “Automated human identification using ear imaging,” Pattern Recognition, vol. 41, no. 5.

[7] Duda, R. O., Hart, P. E., and Stork, D. G. (2001). Pattern Classification. 